\title{
Infant growth and aorta total lipid fatty acids
}

\author{
J Farquharson, E C Jamieson, R W Logan, M B McFadyen, W J A Patrick, A G Howatson, \\ F Cockburn
}

\begin{abstract}
Abnormal fetal and infant growth have increasingly been correlated with adult onset cardiovascular disease. To date, there is little known about the lipid fatty acid profiles in infant cardiovascular tissue. Therefore, we analysed total lipid fatty acids from thoracic and abdominal aorta intima and media from 24 normally grown sudden infant death syndrome cases. Aorta from small for gestational age $(n=2)$, failure to thrive from birth $(n=3)$, and premature $(n=1)$ infants were also examined. Dihomo- $\gamma$-linolenic acid (C20:3n-6) and oleic acid (C18:1n-9) concentrations were significantly lower in the thoracic than in the abdominal aorta. Similar dietary related differences were found in the subgroup $(n=15)$ of infants fed on formula milks. Both abdominal and thoracic intimal arachidonic (C20:4n-6) to dihomo- $\gamma$-linolenic acid ratios were greater in the infants with retarded growth after birth than in their normally grown counterparts. Growth restriction in infancy might disrupt the normal accretion of vascular endothelial polyunsaturated fatty acids.

(Arch Dis Child 1998;79:28-32)
\end{abstract}

Keywords: growth; aorta; fatty acids; formula diet

It has long been postulated that a dietary deficiency of essential fatty acids, linoleic acid (C18:2n-6), $\alpha$-linolenic acid (C18:3n-3), and arachidonic acid (C20:4n-6) might be a predisposing factor in the occurrence of diseases of the cardiovascular system, such as atherosclerosis. ${ }^{1}$ Dietary fatty acid deficiency can cause structural alterations in infant nerve cell membranes, ${ }^{2-4}$ so if such a deficiency is implicated in atheromatous plaque formation, it might cause similar changes in the fatty acid composition of vascular endothelial membranes. ${ }^{5}$

Fatty acids of the aorta, namely dihomo- $\gamma$ linolenic acid (C20:3n-6), arachidonic acid, and eicosapentaenoic acid (C20:5n-3) are important also as parent molecules of the vasoactive eicosanoid series 1, 2, and 3, prostaglandins, prostacyclins $\left(\mathrm{PGI}_{2}\right)$, thromboxanes, and leukotrienes. ${ }^{6}$ Most eicosanoids synthesised from arachidonic acid promote vasoconstriction and increased platelet aggregation.

Some authors think that essential hypertension is predetermined by an adverse fetal environment, ${ }^{78}$ although others believe that there is a contribution from early growth factors affecting weight gain in infancy. ${ }^{9}$ More recently, an association has been found be- tween intrauterine growth retardation and lower diastolic blood pressure at 9 years of age, compared with a cohort of appropriate birthweight. ${ }^{10}$ Arterial eicosanoids cannot be measured in necropsy tissue because of their lability; hitherto, analyses of aorta fatty acids have been confined to small numbers of pooled specimens ${ }^{11}$ or more extensive surveys, which were not linked to either infant diet or growth. ${ }^{12}$ Therefore, we sought to analyse the fatty acids in infant aortic endothelium and to assess the possible significance of their variation with diet, age, growth, and anatomical site.

\section{Subjects and methods}

All subjects had died in the first year of life from sudden infant death syndrome. Aorta $(1-2 \mathrm{~cm})$ was removed at necropsy from both the supradiaphragmatic thoracic and infrarenal abdominal regions and stored intact at $-60^{\circ} \mathrm{C}$ until assay. Cardiac weights were recorded. Gestational age and age at death, together with corresponding birth and body weights, were noted from hospital records and dietary details were obtained from parental interview. Twenty four paired tissues (thoracic and abdominal) were obtained over a three year period (April 1993 to June 1996) from non-growth retarded individuals. ${ }^{13}$ In addition, two paired tissues were obtained from term born, failure to thrive infants, aged 14 and 21 weeks respectively, whose weights (2900 g and $3180 \mathrm{~g}$, respectively) had been above the third centile at birth but had fallen below the first centile at death (4170 $\mathrm{g}$ and $4850 \mathrm{~g}$, respectively), and for which no explanation could be found. We also studied a non-thriving, 11 week old, term infant (3640 $\mathrm{g}$ in weight) with dysmorphic features indicative of fetal alcohol syndrome and evidence of an atrial septal defect. In addition, we retained aorta from two term born infants, aged 5 and 18 weeks, with respective birthweights of $1490 \mathrm{~g}$ and $2130 \mathrm{~g}$, both below the first centile. A 21 week old, preterm infant (26 weeks' gestation) with normal postnatal growth was similarly investigated.

Infants in the normally grown, term born cohort $(n=24)$ were divided into breast fed $(n=7)$ and formula fed groups $(n=15)$. The feeding regimen for two individuals could not be ascertained. Breast fed infants were defined as those who received human milk for at least the first half of their life span. Of the infants not in this main group, only the 14 week old with failure to thrive was breast fed. Unfortunately, because of frequent "unpublicised" alterations to the compositions of formula milks, particularly during the latter stages of this investigation, we could not always assess whether a specific infant formula mentioned in parental
Dr Farquharson.

Accepted 20 January 1998 
Table 1 Thoracic and abdominal aorta intima and media total lipid fatty acids in normally grown term infants

\begin{tabular}{|c|c|c|c|c|}
\hline Fatty acid (wt\%) & Thoracic & Abdominal & $\Delta$ (abdominal - thoracic) & $p$ value ${ }^{\star}$ \\
\hline Palmitic (C16:0) & $25.2(21.9$ to 28.8$)$ & $25.0(19.7$ to 29.0$)$ & $-0.8(-5.0$ to 5.5$)$ & NS \\
\hline Palmitoleic (C16:1n-7) & $1.9(1.1$ to 3.6$)$ & $1.9(0.9$ to 3.8$)$ & $+0.1(-0.4$ to 1.4$)$ & NS \\
\hline Stearic $(\mathrm{C} 18: 0)$ & $17.4(15.2$ to 20.7$)$ & $16.8(15.1$ to 19.1$)$ & $-0.7(-4.2$ to 1.8$)$ & $<0.05$ \\
\hline Oleic $(\mathrm{C} 18: 1 \mathrm{n}-9+\mathrm{n}-7)$ & $20.1(18.4$ to 23.1$)$ & $20.8(18.5$ to 24.4$)$ & $+1.0(-3.1$ to 2.8$)$ & $<0.05$ \\
\hline Linoleic $(C 18: 2 n-6)$ & $7.8(2.6$ to 11.6$)$ & $8.2(3.1$ to 11.8$)$ & $+0.3(-2.2$ to 2.7$)$ & NS \\
\hline Dihomo- $\gamma$-linolenic (C20:3n-6) & $6.4(3.9$ to 8.7$)$ & $7.0(3.4$ to 8.5$)$ & $+0.3(-1.2$ to 2.3$)$ & $<0.05$ \\
\hline Arachidonic (C20:4n-6) & $15.5(13.4$ to 17.9$)$ & $15.2(12.2$ to 18.9$)$ & $-0.6(-2.7$ to 2.2$)$ & NS \\
\hline Docosatetraenoic $(C 22: 4 n-6)$ & $2.9(2.4$ to 4.2$)$ & $3.3(2.3$ to 5.0$)$ & $+0.1(-0.6$ to 1.3$)$ & NS \\
\hline Docosahexaenoic (C22:6n-3) & $2.0(1.3$ to 4.0$)$ & $1.9(1.3$ to 3.6$)$ & $0.0(-0.6$ to 0.7$)$ & NS \\
\hline$\Sigma$ saturated fatty acids & $42.7(39.0$ to 47.0$)$ & $41.5(36.6$ to 46.4$)$ & $-1.0(-6.5$ to 2.4$)$ & NS \\
\hline ¿monounsaturated fatty acids & $22.4(19.8$ to 24.9$)$ & $22.9(20.3$ to 27.3$)$ & $+0.9(-3.0$ to 3.4$)$ & $<0.01$ \\
\hline 乏polyunsaturated fatty acids & $26.6(23.4$ to 32.0$)$ & 26.9 (23.6 to 33.5$)$ & $-0.2(-4.1$ to 5.3$)$ & NS \\
\hline C20:3n-6/C18:2n-6 & $0.78(0.42$ to 1.60$)$ & $0.87(0.46$ to 1.45$)$ & $0.0(-0.6$ to 0.5$)$ & NS \\
\hline$C 20: 4 n-6 / C 20: 3 n-6$ & $2.45(1.74$ to 4.32$)$ & $2.26(1.58$ to 4.60$)$ & $-0.3(-1.0$ to 0.9$)$ & $<0.01$ \\
\hline
\end{tabular}

Infant details-mean birthweight: $3065 \mathrm{~g}$; mean age: 14.4 weeks; age range: 0.5-39 weeks; male to female ratio: 15:9. Results are medians and ranges.

$\Sigma$ polyunsaturated fatty acids $=$ C20:3n-6 + C20:4n-6 + C22:4n-6 +C22:6n-3.

$\star$ Significant differences in fatty acid concentrations and ratios between anatomical sites (thoracic $v$ abdominal) calculated by twotailed Wilcoxon signed rank test for paired data.

interview was devoid of polyunsaturated fatty acids such as dihomo- $\gamma$-linolenic acid, arachidonic acid, and docosahexaenoic acid (C22:6n-3) at that time.

To avoid contamination, adherent muscle, fat, and blood were carefully removed from the adventitia and endothelium and the specimen was weighed. Intima and inner media were stripped from the aorta, weighed and added to $6 \mathrm{ml}$ of chloroform/methanol $(2 / 1 \mathrm{vol} / \mathrm{vol})$ in a glass vial. Because each specimen was localised to a precise aortic site (thoracic or abdominal) and a minimum of $1 \mathrm{~cm}$ of tissue was required, replicate sampling was not possible. The inner aspect of the artery was chosen for fatty acid analysis because this area is invaded by atherosclerotic plaque formation in early life. ${ }^{14}$ The tissue was ultrasonicated (Soniprep 150 ultrasonicator; MSE, London, UK) for $4 \times 6$ seconds at an amplitude of $4 \mu \mathrm{m}$. Lipids isolated were weighed, solubilised in $200 \mu \mathrm{l}$ chloroform, and derivatised with $2 \mathrm{ml}$ boron trifluoride/methanol ( $14 \% \mathrm{wt} / \mathrm{vol})$, as described previously. ${ }^{2}$ The fatty acid methyl esters formed were separated by gas chromatography on a $25 \mathrm{~m}$ fused silica column $(0.53 \mathrm{~mm}$ internal diameter) with non-polar BPX1 stationary phase (SGE Ltd, Victoria, Australia). An initial column temperature of $180^{\circ} \mathrm{C}$ was held for two minutes, then increased to $280^{\circ} \mathrm{C}$ by $5^{\circ} \mathrm{C} /$ minute, and injection and detector temperatures were set at $300^{\circ} \mathrm{C}$

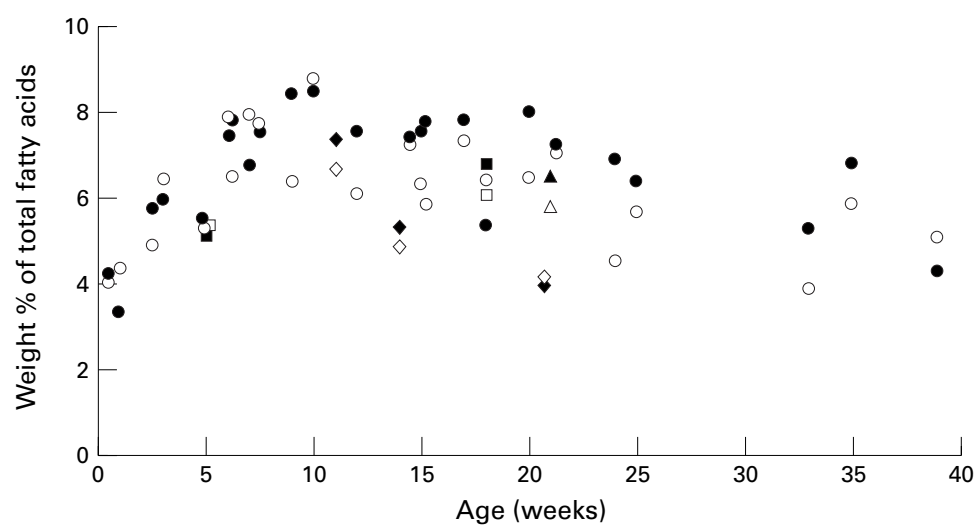

Figure 1 Abdominal and thoracic aorta intima and media dihomo- $\gamma$-linolenic acid (C20:3n-6) in relation to the age and growth of infants. Filled symbols, abdominal aorta; open symbols, thoracic aorta. Circles, normally grown infants; squares, small for gestational age infants; diamonds, failure to thrive (after birth) infants; triangles, preterm infants. and $350^{\circ} \mathrm{C}$, respectively. The gas chromatograph output was linked to a computing integrator (CDP1-Pye Unicam; Pye Unicam, Cambridge, UK) to calculate peak areas produced by the individual fatty acid methyl esters. A commercial, gravimetric standard solution (Supelco 37; Sigma Chemical Co, Poole, Dorset, UK) containing known amounts of fatty acid methyl esters in varying weight ratios was analysed to verify the uniformity of detector (flame ionisation) response.

Nine major fatty acids were identified by their column retention times with reference to authentic individual standards (Sigma Chemical Co) and concentrations expressed as weight percentages of total fatty acids. Only results from normally grown individuals are presented in tables 1 and 2, the latter composed of dietary dependent (human or formula milk) subgroups. Also shown are total saturated, monounsaturated and polyunsaturated fatty acids (excluding linoleic acid) together with ratios of essential fatty acids and their products. The distribution of results with age, including those from the small for gestational age, failure to thrive, and premature infants are given for dihomo- $\gamma$-linolenic (fig 1), arachidonic acid (fig 2), and the arachidonic acid/dihomo- $\gamma$ linolenic ratio (fig 3).

\section{STATISTICAL ANALYSIS}

Differences in fatty acid concentrations between anatomical sites (thoracic and abdominal) were assessed, irrespective of diet, for the normally grown infants $(n=24)$ by the two tailed Wilcoxon's signed rank test for paired data (table 1). Low numbers precluded statistical analysis of the small for gestational age $(\mathrm{n}=2)$ and non-thriving $(\mathrm{n}=3)$ infants with respect to the normally grown cohort. However, their fatty acid concentrations were evaluated with respect to age comparable individuals from within that group. Because the feeding groups (human versus formula milk) were not age matched, the effect of diet on aorta fatty acid compositions was limited to between site comparisons within each dietary group and differences were assessed as before by the Wilcoxon signed rank test (table 2). 


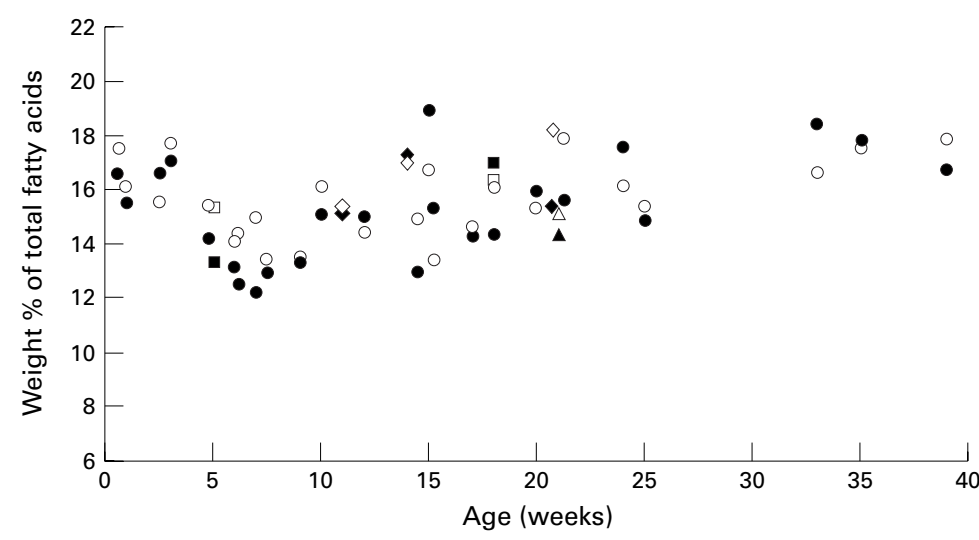

Figure 2 Abdominal and thoracic aorta intima and media arachidonic acid (C20:4n-6) in relation to the age and growth of infants. Filled symbols, abdominal aorta; open symbols, thoracic aorta. Circles, normally grown infants; squares, small for gestational age infants; diamonds, failure to thrive (after birth) infants; triangles, preterm infants.

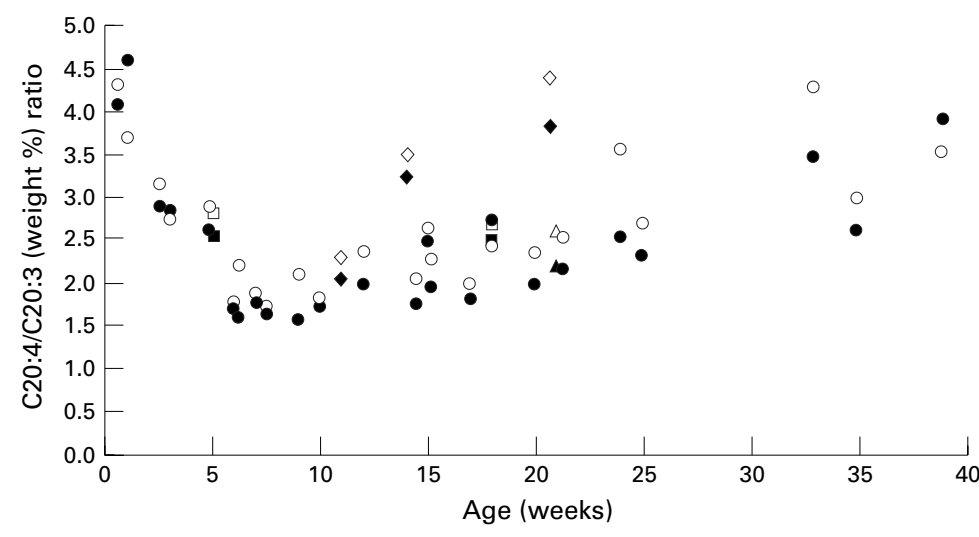

Figure 3 Abdominal and thoracic aorta intima and media arachidonic acid (C20:4n-6)/ dihomo- $\gamma$-linolenic acid $(C 20: 3 n-6)$ ratio in relation to age and growth of infants. Filled symbols, abdominal aorta; open symbols, thoracic aorta. Circles, normally grown infants; squares, small for gestational age infants; diamonds, failure to thrive (after birth) infants; triangles, preterm infants.

\section{Results}

Mean (SD) recoveries of abdominal and thoracic intima and media from the aorta at $27.3 \%(6.2 \%)$ and $26.0 \%(6.1 \%)$, respectively, were not significantly different. In the growth retarded infants, organ weights were commensurate with reduced body weight.

Application of the Wilcoxon signed rank test to the paired data (table 1) showed significantly higher stearic acid (C18:0) concentrations in the thoracic tissue $(\mathrm{p}<0.05)$, whereas significantly higher concentrations of oleic acid (C18:1) were found in abdominal tissue $(\mathrm{p}<0.05)$. The trend in dihomo- $\gamma$-linolenic acid results was for an initial postnatal rise from $\sim 4 \%$ to a maximum of $\sim 9 \%$ at 10 weeks, followed by a slow decline thereafter (fig 1). In contrast, arachidonic acid concentrations fell from $\sim 17 \%$ at birth to a minimum of $12 \%$ at 8 weeks, with a subsequent gradual increase (fig 2). A similarly shaped curve with a minimum at $2.3 \%$ was obtained for docosatetraenoic acid (C22:4n-6). Overall, the C20:4/C20:3 ratio (as a possible measure of $\Delta 5$ desaturase enzyme activity) displayed an initial reduction to a minimum at 9 weeks, which was also reversed with time (fig 3). Within these data, dihomo- $\gamma-$ linolenic acid was significantly lower in the thoracic intima $(p<0.05)$, whereas arachidonic acid was higher, although the difference did not reach significance (table 1). As a consequence, C20:4/C20:3 ratios were significantly greater in the thoracic group than in the abdominal aorta group ( $\mathrm{p}<0.01)$ (table 1$)$. No between group differences were apparent for either docosatetraenoic or docosahexaenoic acid, although the lowest concentrations of docosahexaenoic acid were detected in the thoracic $(1.4 \%)$ and abdominal intima $(1.1 \%)$ from the 21 week old formula fed preterm infant.

In the failure to thrive cases, the 14 and 21 week old infants had apparently "low" dihomo$\gamma$-linolenic acid concentrations when compared with their normally grown contemporaries (fig 1), which in combination with equivalent arachidonic acid concentrations (fig 2) resulted in relatively higher $C 20: 4 / C 20: 3$ ratios in all tissues from these growth retarded infants (fig 3). No similar effect was evident in the results of the preterm and small for gestational age individuals who maintained aorta intimal fatty acid compositions that were indistinguishable from those of appropriately developed infants of similar age (figs 1 and 2).

When the effect of diet was considered (Wilcoxon), no significant differences in aorta fatty acid concentrations between anatomical sites

Table 2 Thoracic and abdominal aorta intima and media total fatty acids in relation to infants' diet

\begin{tabular}{|c|c|c|c|c|c|c|c|}
\hline \multirow[b]{2}{*}{ Fatty acid } & \multicolumn{3}{|l|}{ Human milk } & \multicolumn{4}{|l|}{ Formula milk } \\
\hline & Abdominal & Thoracic & $\begin{array}{l}\Delta \text { (abdominal - } \\
\text { thoracic) }\end{array}$ & Abdominal & Thoracic & $\begin{array}{l}\Delta \text { (abdominal - } \\
\text { thoracic) }\end{array}$ & $p$ value ${ }^{\star}$ \\
\hline C16:0 & 25.1 (22.8 to 29.0$)$ & 25.5 (21.9 to 28.8$)$ & $+0.6(-2.8$ to 5.5$)$ & 24.9 (19.7 to 28.6$)$ & 25.6 (23.7 to 28.6$)$ & $-1.1(-5.0$ to 1.5$)$ & NS \\
\hline C16:1 & $2.5(1.6$ to 3.8$)$ & $2.3(1.4$ to 3.6$)$ & $+0.2(-0.4$ to 1.4$)$ & $1.8(0.9$ to 3.4$)$ & $1.9(1.1$ to 2.6$)$ & $0.0(-0.3$ to 0.8$)$ & NS \\
\hline C18:0 & 16.5 (15.9 to 18.1$)$ & $17.4(16.0$ to 20.7$)$ & $-1.1(-4.2$ to 1.4$)$ & $16.8(15.1$ to 19.1$)$ & $17.2(15.2$ to 18.6$)$ & $-0.5(-2.1$ to 1.8$)$ & NS \\
\hline C18:1 & $20.6(19.7$ to 22.1$)$ & 20.3 (18.4 to 22.2$)$ & $+0.9(-1.9$ to 1.7$)$ & $21.1(19.5$ to 23.7$)$ & $19.7(18.4$ to 23.1$)$ & $+1.1(-3.2$ to 2.7$)$ & $<0.05$ \\
\hline $\mathrm{C} 18: 2$ & $5.8(3.1$ to 11.8$)$ & $6.7(2.6$ to 11.1$)$ & $+0.5(-1.9$ to 1.6$)$ & $8.5(6.8$ to 11.6$)$ & $8.6(6.5$ to 11.6$)$ & $-0.6(-2.2$ to 2.7$)$ & NS \\
\hline $\mathrm{C} 20: 3$ & $5.7(3.4$ to 8.5$)$ & $6.4(4.1$ to 8.7$)$ & $-0.3(-1.0$ to 2.0$)$ & $7.4(4.2$ to 8.0$)$ & $6.3(3.9$ to 7.9$)$ & $+0.9(-1.2$ to 2.3$)$ & $<0.02$ \\
\hline $\mathrm{C} 20: 4$ & $15.5(13.3$ to 17.1$)$ & $16.1(13.5$ to 17.7$)$ & $-0.7(-1.7$ to 1.1$)$ & $15.1(12.2$ to 18.9$)$ & $15.0(13.4$ to 17.9$)$ & $-0.3(-2.7$ to 2.2$)$ & NS \\
\hline $\mathrm{C} 22: 4$ & $3.4(2.9$ to 3.7$)$ & $2.9(2.4$ to 4.2$)$ & $+0.1(-0.6$ to 0.9$)$ & $3.4(2.3$ to 5.0$)$ & $2.9(2.4$ to 4.2$)$ & $+0.1(-0.6$ to 1.3$)$ & NS \\
\hline C22:6 & $2.4(2.0$ to 3.6$)$ & $2.7(2.0$ to 4.0$)$ & $+0.2(-0.6$ to 0.6$)$ & $1.7(1.3$ to 2.7$)$ & $1.7(1.3$ to 2.4$)$ & $0.0(-0.5$ to 0.7$)$ & NS \\
\hline$\Sigma$ sat'd FA & 42.6 (38.6 to 45.5$)$ & 42.5 (39.0 to 47.0$)$ & $+0.7(-3.9$ to 1.4$)$ & 41.5 (36.6 to 46.4$)$ & 42.9 (39.9 to 45.7$)$ & $-1.2(-6.5$ to 2.4$)$ & $<0.05$ \\
\hline ¿monosat'd FA & 23.0 (21.3 to 25.9$)$ & $23.3(19.8$ to 24.9$)$ & $+0.5(-1.8$ to 2.0$)$ & $22.9(20.3$ to 25.8$)$ & 21.3 (20.1 to 24.7$)$ & $+0.9(-3.0$ to 2.7$)$ & $<0.05$ \\
\hline Epolysat'd FA & $27.0(24.9$ to 30.1$)$ & $26.6(24.3$ to 32.0$)$ & $-1.6(-2.4$ to 3.4$)$ & 26.8 (23.6 to 33.5$)$ & $26.2(23.4$ to 29.6$)$ & $-0.1(-4.1$ to 5.3$)$ & NS \\
\hline C20:3/C18:2 & $0.88(0.48$ to 1.45$)$ & $0.89(0.58$ to 1.58$)$ & $-0.15(-0.6$ to 0.5$)$ & $0.90(0.46$ to 1.10$)$ & $0.66(0.42$ to 1.11$)$ & $+0.06(-0.2$ to 0.4$)$ & NS \\
\hline C20:4/C20:3 & $2.86(1.58$ to 4.60$)$ & $2.75(1.84$ to 4.32$)$ & $-0.06(-0.5$ to 0.9$)$ & $2.00(1.61$ to 3.94$)$ & $2.37(1.74$ to 4.31$)$ & $-0.33(-1.0$ to 0.4$)$ & $<0.01$ \\
\hline
\end{tabular}

Infant details—mean birthweight: human milk $3325 \mathrm{~g}$, formula $2960 \mathrm{~g}$; mean age: human milk 6.3 weeks, formula 18.5 weeks; age range: human milk 0.5-18 weeks, formula 6-39 weeks; male to female ratio: human milk 3:4, formula 11:4.

Results are medians and ranges and expressed as weight percentage of total fatty acids.

* Significant differences in fatty acid concentrations (Wilcoxon signed rank test) between anatomical sites (thoracic $v$ abdominal) are given for the formula fed group only. No significant differences were found in the human milk fed infants. 
were revealed in the breast fed $(n=7)$ subgroup (table 2). Within the formula fed group $(n=15)$, differences in fatty acid concentrations were similar to those of the total group (table 1), with the exception that the significantly higher thoracic aorta stearic acid (C18:0) concentration was substituted by that for total saturated fatty acids (table 2 ).

\section{Discussion}

The low recoveries of aorta intima total lipid (generally $<1 \%$ of tissue wet weight) might indicate that the primary function of these fats in this tissue is not a structural one. Their importance might be a result of their role as eicosanoid precursors. Eicosanoid metabolism is thought to be governed by the availability of the precursor (C20) fatty acids released by phospholipase $\mathrm{A}_{2}$ hydrolysis of the parent membrane phospholipid. Eicosanoids work over a short range at very low concentrations, and those derived from arachidonic acid are vastly more potent mediators of vasoactive and thrombotic processes than those derived from dihomo- $\gamma$-linolenic acid. Our results demonstrate a significantly lower ratio of arachidonic acid to dihomo- $\gamma$-linolenic acid in the abdominal than the thoracic intima. Also, the initial fall in both arachidonic acid concentrations and arachidonic acid:dihomo- $\gamma$-linolenic acid ratios (figs 2 and 3) parallels that of early infant diastolic blood pressure, which is the only period in normal human development when such a reduction in blood pressure is experienced. ${ }^{15}$

Aorta dihomo- $\gamma$-linolenic acid concentrations in the immediate postnatal period (5-20 weeks) of $\sim 8 \%$ of the total lipid fatty acids (fig 1) are greater than has been encountered previously in other human tissues. However, at 10 weeks the arachidonic acid:dihomo- $\gamma$ linolenic acid ratio reaches a minimum of $\sim 1.5$ (fig 3) and thereafter increases throughout the first year of life as a reversal in arachidonic acid and dihomo- $\gamma$-linolenic acid concentrations occurs. The intima arachidonic acid composition reached by 40 weeks is then maintained throughout life, although a further reduction occurs in dihomo- $\gamma$-linolenic acid concentrations, resulting in arachidonic acid/dihomo- $\gamma$ linolenic acid ratios of $\sim 10$ throughout adult life. ${ }^{12}$ Although inadequate weight gain in the first years of life has been postulated as a predictor of increased susceptibility to hypertension in later life, ${ }^{9}$ no causative factor has yet been identified. The two older infants that we studied with failure to thrive from birth had greater ratios of aortic intimal arachidonic acid to dihomo- $\gamma$-linolenic acid than their normally grown counterparts (fig 3), which appeared to be largely because of a reduced incorporation of dihomo- $\gamma$-linolenic acid (fig 1). No such effect was found in the 21 week old preterm infant (26 weeks' gestation), whose aorta fatty acid concentrations seemed appropriate for his chronological age (figs 1 and 2). However, the accompanying general physical dimensions of this infant's aorta and cardiac weight $(20 \mathrm{~g})$, as expected, correlated more closely with postconceptual age. The apparent ability to accrete polyunsaturated fatty acids during fetal life is seen in the intrauterine growth retarded infants (figs 1 and 2), in whom concentrations were indistinguishable from those of normally grown neonates. Dietary related differences in aorta fatty acid concentrations between the supradiaphragmatic and infrarenal sites were restricted to the infants fed on formula milks devoid of dihomo- $\gamma$-linolenic acid, arachidonic acid, and docosahexaenoic acid. However, the breast fed group was small and contained a disproportionate number of younger individuals (table 2). Any discussion of aorta fatty acids must recognise that the total endogenous lipid content of the infant aorta vessel wall is minimal $(<500 \mathrm{mg}$ ), although the lowest concentrations of docosahexaenoic acid were recorded in the intima of the formula fed premature infant.

The relative differences in saturated (stearic) and monounsaturated (oleic) fatty acids between anatomical sites (tables 1 and 2) probably reflects a marginally greater triglyceride (+ cholesterol ester) to phospholipid ratio in the abdominal vasculature because, in general, the highest concentrations of oleic acid are located in the triglyceride ${ }^{16}$ and cholesterol esters. ${ }^{11}$ This increased incorporation of mono-unsaturated oleic acid is usually at the expense of saturated fatty acids and might explain why diets rich in oleic acid content appear to confer a protective effect against heart disease by lowering low density lipoprotein cholesterol. ${ }^{17} 18$

As far as we are aware, clinical outcome in terms of cardiovascular development has not been studied specifically in a non-thriving infant population and, therefore, the possible implications of variations in arachidonic acid:dihomo- $\gamma$-linolenic acid ratios are unknown. We have found lower arachidonic acid:dihomo- $\gamma$-linolenic ratios in abdominal than thoracic aorta intima, and identified that the reduction in arachidonic acid is concurrent with that of diastolic blood pressure in the first months of life. However, the properties of newly developing, more peripheral vessels (arterioles and capillaries) might be influenced by fatty acid compositions different from those found in the aorta. If the "reduced" concentrations of dihomo- $\gamma$-linolenic acid in the failure to thrive infant aortas are pathogenic and indicative of increased $n-6$ series $\Delta 5$ desaturase activity then dietary manipulation by increasing the intake of $n-3$ series fatty acids might correct the "abnormality" by providing competitive inhibition of the enzyme system.

1 Sinclair HM. Deficiency of essential fatty acids and artherosclerosis, etcetera. Lancet 1956;i:381-3.

2 Farquharson J, Cockburn F, Patrick WJA, et al. Infant cerebral cortex phospholipid fatty acid composition and diet. Lancet 1992;340:810-13.

3 Makrides M, Neumann MA, Byard RW, et al. Fatty acid composition of brain, retina and erythrocytes in breast- and formula-fed infants. Am $\mathcal{F}$ Clin Nutr 1994;60:189-94.

4 Farquharson J, Jamieson EC, Logan RW, et al. Docosahexaenoic and nervonic acids in term and preterm infant cerebral white matter. Prenat Neonat Med 1996;1:234-40.

5 Bottcher CJF, Woodford FP, Romeny-Wachter CCTH, et al. Fatty-acid distribution in lipids of the aortic wall. Lancet 1960;ii:1378-83.

6 Needleman P, Turk J, Jakschik BA, et al. Arachidonic acid metabolism. Annu Rev Biochem 1986;55:69-102. 
7 Barker DJP, Gluckman PD, Godfrey KM, et al. Fetal nutrition and cardiovascular disease in adult life. Lancet tion and cardiovas

8 Law CM, de Swiet M, Osmond C, et al. Initiation of hypertension in utero and its amplification throughout life. $B M \mathcal{F}$ 1993;306:24-7.

9 Whincup PH, Cook DG, Shaper AG. Early influences on blood pressure: a study of children aged 5-7 years. $B M \mathcal{F}$ 1989;299:587-91.

10 Ley D, Stale H, Marsal K. Aortic vessel wall characteristics and blood pressure in children with intrauterine growth retardation and abnormal foetal aortic blood flow. Acta Paediatr 1997;86:299-305.

11 Day AJ, Wahlqvist ML. Cholesterol ester and phospholipid composition of normal aortas and of atherosclerotic lesions in children. Exp Mol Pathol 1970;13:199-216.

12 Wiese HF, Coon E, Yamanaka W, et al. Lipid composition of the vascular system during infancy, childhood, and young

13 Tanner JM, Whitehouse RH, Takaishi M. Standards from birth to maturity for height, weight, height velocity and weight velocity; British children, 1965. Arch Dis Child 1966;41:613-35.

14 Stary HC. The sequence of cell and matrix changes in atherosclerotic lesions of coronary arteries in the first forty years of life. Eur Heart f 1990;11 (suppl E):3-19.

15 Report from the second task force on blood pressure control in children. Paediatrics 1987;79:1-25.

16 Farquharson J, Cockburn F, Patrick WJA, et al. Effect of diet on infant subcutaneous tissue triglyceride fatty acids. Arch Dis Child 1993;69:589-93.

17 NCEP (National Cholesterol Education Program). Second report of the expert panel on detection, evaluation and treatment of high blood cholesterol in adults. Circulation 1994;89:1329-445.

18 Superko HR, Krauss RM. Coronary artery disease regression. Convincing evidence for the benefit of aggressive lipoprotein management. Circulation 1994;90:105669.

\section{Frey's syndrome in infancy}

Here is a diagnosis that will amaze your peers and make you the (temporary) star of the clinical meeting. Have you ever come across Frey's syndrome (auriculotemporal nerve syndrome) in infancy?

It is a syndrome with a history; first described by Duphenix in 1757 but rediscovered by Frey in 1923. Mostly seen in adults with problems in or around the parotid gland, it occurs rarely in children and then usually soon after the introduction of solid foods. American and Australian clinicians have recently described eight children who developed symptoms in infancy (Maria Victoria Dizon and colleagues; Archives of Dermatology 1997;133:1443-5). That is, they developed the symptom localised facial erythema almost immediately after tasting solid foods and lasting for 30 to 60 minutes. Unlike adults, the children did not exhibit sweating over the affected area of skin. Photographs of two of the children show two areas of erythema, one in the cutaneous distribution of the auriculotemporal nerve in front of the upper part of the pinna, and one in the centre of the cheek, in the area of the maxillary branch of the trigeminal nerve (should it be auriculotemporotrigeminal syndrome?). It is said that the flushing often diminishes with time; it did so before the age of 5 years in three of six children followed up in this series. Two of the eight children had bilateral flushing. The condition in infants is usually attributed to forceps injury, and six of the eight had forceps delivery. Treatment is unnecessary.

I'm not fond of telephone diagnosis but perhaps you could add this to your list of conditions that could be diagnosed that way.

ARCHIVIST 